\title{
P87 Ultra-high Frequency Ultrasound Assessment in Vascular Ehlers Danlos Syndrome: a Validation and Reproducibility Study
}

\author{
Rosa Maria Bruno ${ }^{1,2}$, Magdalini Alexopoulou ${ }^{1}$, Hakim Khettab ${ }^{3}$, Xavier Jeunemaitre ${ }^{3}$ Pierre Boutouyrie $^{3}$ \\ ${ }^{1}$ University of Pisa, Pisa, Italy \\ ${ }^{2}$ INSERM U970, Equipe 7, Paris, France \\ ${ }^{3}$ APHP, Hôpital Européen Georges Pompidou, Paris, France
}

\section{ABSTRACT}

Background: In patients with Vascular Ehlers-Danlos syndrome (vEDS), an abnormally low carotid intima-media thickness (CIMT) may increase the risk of arterial dissection and rupture. Thus its accurate assessment by ultra-high frequency ultrasound (UHFUS), thanks to its higher spatial resolution, may be clinically relevant.

Aim: To assess the feasibility and reproducibility of carotid parameters, assessed by UHFUS in vESD patients, and to evaluate the agreement with the gold standard technique, echotracking by radiofrequency.

Methods: 16 vEDS patients were recruited (6 women, $40 \pm 11$ years, BP $115 \pm 6 / 62 \pm 6 \mathrm{mmHg}$ ). Common carotid parameters were assessed by echotracking (Esaote, Artlab software) and UHFUS (VevoMD, Visualsonics; CVSuite software, Quipu srl), to evaluate agreement. The coefficient of variation between two consecutive clips was computed. The results were compared to those of 16 age-, sex- and BP-matched healthy individuals.

Results: In all 16 patients the acquisition and automated analysis of carotid clips was feasible. Correlation between echotracking and UHFUS was satisfactory (diameter $r=0.63, p=0.001$; CIMT $r=0.65, p=0.006$; distension $r=0.84, p<0.001$ ). BlandAltman plots showed a good agreement between the two techniques, with a non significant bias either for diameter [110 $\mu \mathrm{m}$ $(-184 ; 404)$ ] or CIMT [27 $\mu \mathrm{m}(-10 ; 75)]$. Intra-operator coefficient of variation was $3.26 \%$ (diameter), $7.11 \%$ (CIMT) and 5.65\% (distension). vEDS patients had reduced CIMT ( $419 \pm 85$ vs $522 \pm 97 \mu \mathrm{m}, p=0.004)$ and distension (453 \pm 150 vs $613 \pm 176 \mu \mathrm{m}$, $p=0.01$ ) than controls and tended to have a reduced diameter (6558 $\pm 525 \mathrm{vs} 6945 \pm 653 \mu \mathrm{m}, p=0.08$ ), while carotid-femoral pulse wave velocity was similar $(7.38 \pm 1.08$ vs $7.46 \pm 1.396 \mathrm{~m} / \mathrm{s}, p=0.78)$.

Conclusion: UHFUS is feasible, accurate and reproducible for the evaluation of carotid parameters in vEDS.

(c) 2019 Association for Research into Arterial Structure and Physiology. Publishing services by Atlantis Press International B.V.

This is an open access article distributed under the CC BY-NC 4.0 license (http://creativecommons.org/licenses/by-nc/4.0/). 